\title{
Stellar activity and magnetic shielding
}

\author{
J.-M. Grießmeier ${ }^{1}$, M. Khodachenko ${ }^{2}$, H. Lammer ${ }^{2}$, J. L. Grenfell ${ }^{3}$, \\ A. Stadelmann ${ }^{4}$ and U. Motschmann ${ }^{4}$ \\ ${ }^{1}$ ASTRON, Postbus 2, 7990 AA, Dwingeloo, The Netherlands \\ email: griessmeier@astron.nl \\ ${ }^{2}$ Space Research Institute, Austrian Academy of Sciences, Schmiedlstr. 6, A-8042 Graz, Austria \\ ${ }^{3}$ Zentrum für Astronomie und Astrophysik, Technische Universität Berlin (TUB), \\ Hardenbergstr. 36, 10623 Berlin, Germany \\ ${ }^{4}$ Technical University of Braunschweig, Mendelssohnstraße 3, 38106 Braunschweig, Germany
}

\begin{abstract}
Stellar activity has a particularly strong influence on planets at small orbital distances, such as close-in exoplanets. For such planets, we present two extreme cases of stellar variability, namely stellar coronal mass ejections and stellar wind, which both result in the planetary environment being variable on a timescale of billions of years. For both cases, direct interaction of the streaming plasma with the planetary atmosphere would entail servere consequences. In certain cases, however, the planetary atmosphere can be effectively shielded by a strong planetary magnetic field. The efficiency of this shielding is determined by the planetary magnetic dipole moment, which is difficult to constrain by either models or observations. We present different factors which influence the strength of the planetary magnetic dipole moment. Implications are discussed, including nonthermal atmospheric loss, atmospheric biomarkers, and planetary habitability.
\end{abstract}

Keywords. astrobiology, planetary systems, magnetic fields, stars: winds, outflows, Sun: coronal mass ejections (CMEs), cosmic rays

\section{Introduction}

One of the many fascinating questions in the field of exoplanet studies is the search for habitable worlds. Because of their relatively small mass, low luminosity, long lifetime and large abundance in the galaxy, $\mathrm{M}$ dwarfs are sometimes suggested as prime targets in searches for terrestrial habitable planets (Tarter et al. 2007; Scalo et al. 2007). Interestingly, M dwarfs also seem to have a larger number of Super-Earth planets (i.e. planets with a mass smaller than 10 terrestrial masses, i.e. $M \lesssim 10 M_{\mathrm{E}}$ ) than more massive stars (Forveille et al. 2009). Excluding planets orbiting around pulsars, the first super-Earth detected was GJ $876 \mathrm{~d}$, a planet with $\sim 7.5 M_{\mathrm{E}}$ orbiting an M star (Rivera et al. 2005). Thereafter, other super-Earth planets have been discovered (Beaulieu et al. 2006; Lovis et al. 2006; Udry et al. 2007; Ribas et al. 2008; Mayor et al. 2009; Forveille et al. 2009; Howard et al. 2009; Bouchy et al. 2009). In total, 12 planets with masses $\lesssim 10 M_{\mathrm{E}}$ are reported today, and more detections are expected for the near future (Howard et al. 2009; Mayor et al. 2009)†. The least massive planet has a mass of $4.2 M_{\mathrm{E}}$.

For M stars the habitable zone (HZ, defined by the range of orbital distances over which liquid water is possible on the planetary surface, see e.g. Kasting et al. 1993) is much closer to the star (depending on stellar mass, but typically $\leqslant 0.3$ AU). Such close-in distances pose unique problems and constraints to habitability. For example, such planets are exposed to strong stellar winds in the early phases of the host star's evolution. Also, one

$\dagger$ Up-to-date numbers can be found at the Extrasolar Planets Encyclopaedia: http://www . exoplanet.eu 
expects a large number of coronal mass ejections (CMEs) on active M stars (Houdebine et al. 1990; Scalo et al. 2007). The related interaction of the dense plasma flux (either from the stellar wind or a CME) with the atmosphere/magnetosphere environment of the exposed planets during the active stage of the stellar evolution could be strong enough to erode the atmosphere or the planets' water inventory via non-thermal atmospheric loss processes (Khodachenko et al. 2007; Lammer et al. 2007). Weak magnetic shielding of tidally locked planets will also lead to an increased influx of galactic cosmic rays and stellar energetic particles (Grießmeier et al. 2005, 2009), which can have important consequences for biological systems, both directly and indirectly via the modification of the planetary atmosphere (e.g. destruction of atmospheric ozone, see Grenfell et al. 2007, 2010).

This paper is organised as follows: The evolution of the conditions in the stellar vicinity over the timescale of the stellar evolution is presented in Section 2. Two cases are considered: stellar CMEs (Section 2.1), and the average stellar wind (Section 2.2). Planetary magnetic shielding against the influence of the star is discussed in Section 3. Consquences are discussed in Section 4, with special focus on the potential evaporation of the planetary atmosphere (Section 4.1) and the flux of cosmic rays to the planetary atmosphere (Section 4.2). Section 5 closes with a few concluding remarks.

\section{Stellar variability}

Stars are known to be variable in may different ways and on many different timescales. Effects such as stellar CMEs, flares, starspots and stellar rotation occur on timescales of hours or days, while stellar magnetic cycles are expected on the timescale of decades or even centuries. Stellar irradiation, the stellar CME activity and the stellar wind parameters evolve very slowly over Gyr. Many of these effects are discussed elsewhere in this volume. In this section we focus on the slow evolution of two of the stellar parameters over the stellar evolutionary timescale, namely the evolution of stellar CME parameters and of the average stellar wind.

\subsection{Stellar CMEs}

There are two reasons why in addition to the steady stellar wind CMEs have to be taken into account for the interaction with the planetary atmosphere: (a) Due to their limited propagation distance, CMEs are more frequent for close-in exoplanets than at larger distances, and (b) CMEs can be much denser and faster than the average stellar wind, thus exerting a considerable influence on the planet.

At 1 AU, CME collisions with the terrestrial magnetosphere are relatively rare events. However, this is due to the fact that most CMEs cannot be detected at orbital distances $\geqslant 0.1$ AU. Only $\leqslant 20 \%$ of CMEs can reach distances of $\approx 1$ AU and more. Thus, for a planet at $0.1 \mathrm{AU}$, where all CMEs have to be taken into account, the number of CMEplanet collisions is expected to be considerably higher (Khodachenko et al. 2007).

An estimation of CME parameters (combining in-situ measurements near the sun (e.g. by Helios) with remote solar observation by SoHO, Khodachenko et al. 2007) has shown that for small orbital distances, CMEs can be considerably denser and faster than the solar wind (roughly one order of magnitude denser and half an order of magnitude faster). Here, we assume that stellar CMEs behave similarly to solar ones ("solar-stellar analogy", see Khodachenko et al. 2007). If CME-planet collisions are frequent enough (such that the planet effectively moves through a constant background of CME plasma), the interaction of the planetary atmosphere with stellar CMEs can be estimated by replacing the stellar wind parameters $n(d)$ and $v(d)$ by the corresponding parameters 
$n_{\mathrm{CME}}(d)$ and $v_{\mathrm{CME}}(d)$ for strong CMEs as given by Khodachenko et al. (2007). The consequences for atmospheric erosion will be discussed in Section 4.1.

\subsection{Stellar wind}

The stellar wind density $n$ and velocity $v$ encountered by a planet are key parameters defining the size of the magnetosphere. As these stellar wind parameters depend on the stellar age, the quality of magnetic shielding is strongly time dependent.

For stellar ages $>0.7 \mathrm{Gyr}$, the radial dependence of the stellar wind properties is well described by a Parker-like (Parker 1958) stellar wind model. In this model, the interplay between stellar gravitation and pressure gradients leads to a supersonic gas flow for sufficiently large substellar distances $d$. The free parameters are the coronal temperature and the stellar mass loss. They are indirectly chosen by setting the stellar wind conditions at 1 AU. More details on the model can be found elsewhere (e.g. Parker 1958; Mann et al. 1999; Prölss 2004; Grießmeier et al. 2007).

The dependence of the stellar wind density $n$ and velocity and $v$ on the age of the stellar system is measured by observing astrospheric absorption features of stars with different ages. The characteristic Ly $\alpha$ absorption feature (at $1216 \AA$ ) created by neutral hydrogen at the astropause was detected in high-resolution observations obtained by the Hubble Space Telescope (HST). Comparing the measured absorption to that calculated by hydrodynamic codes, these measurements allowed the first empirical estimation of the evolution of the stellar mass loss rate as a function of stellar age (Wood et al. 2002; Wood 2004; Wood et al. 2005). It should be noted, however, that the resulting estimates are only valid for stellar ages $t \geqslant 0.7 \mathrm{Gyr}$ (Wood et al. 2005). These results for the mass loss rate can be combined with the model for the age-dependence of the stellar wind velocity of Newkirk (1980) to obtain the age-dependent stellar wind density. One thus finds (Grießmeier et al. 2007):

$$
v(1 \mathrm{AU}, t)=v_{0}\left(1+\frac{t}{\tau}\right)^{-0.43},
$$

and

$$
n(1 \mathrm{AU}, t)=n_{0}\left(1+\frac{t}{\tau}\right)^{-1.86 \pm 0.6},
$$

with $v_{0}=3971 \mathrm{~km} / \mathrm{s}, n_{0}=1.04 \cdot 10^{11} \mathrm{~m}^{-3}$ and $\tau=2.56 \cdot 10^{7} \mathrm{yr}$. The dependence of $n(1 \mathrm{AU}, t)$ and $v(1 \mathrm{AU}, t)$ as a function of stellar age is shown in Figs. 1(a) and 1(b) for the case of a sun-like star.

The procedure to obtain the stellar wind velocity $v\left(d, M_{\star}, R_{\star}\right)$ and density $n\left(d, M_{\star}, R_{\star}\right)$ at the location of an exoplanet (i.e. at distance $d$ ) for a host star of given mass $M_{\star}$, and radius $R_{\star}$ and age $t$ from $v(1 \mathrm{AU}, t)$ and $n(1 \mathrm{AU}, t)$ is described in Grießmeier et al. (2005, 2009).

\section{Magnetic shielding}

For an efficient magnetic shield to exist, the planet requires a strong magnetic field. A necessary condition for a magnetic dynamo is a large, electrically conducting fluid region in non-uniform motion (i.e. the liquid outer core for terrestrial planets, or a layer of electrically conducting hydrogen for gas giants). According to planetary dynamo theory, this flow should be convective in nature (Stevenson 1983). In practice, all planets with core convection can be assumed to have a magnetic dynamo (Stevenson 1983). Conversely, convection can be regarded as a necessary requirement for a planetary magnetic field. 


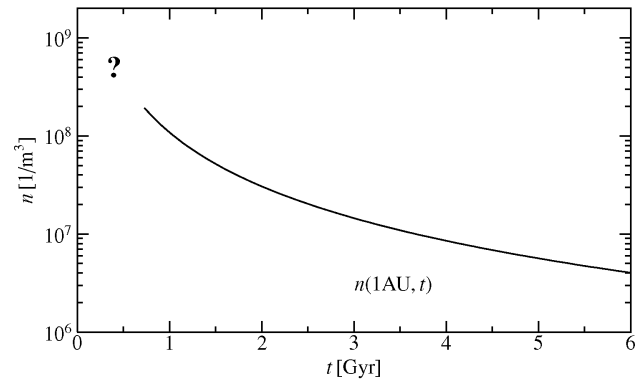

(a) Stellar wind density $n(1 \mathrm{AU}, t)$.

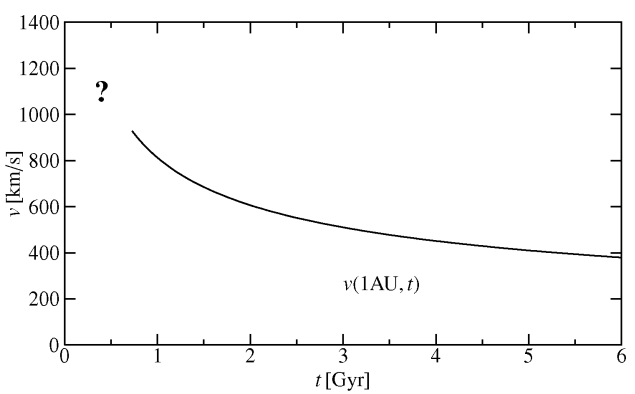

(b) Stellar wind velocity $v(1 \mathrm{AU}, t)$.

Figure 1. Stellar wind density $n(t)$ and velocity $v(t)$ at $1 \mathrm{AU}$ distance of a sun-like star $\left(M_{\star}=M_{\odot}, R_{\star}=R_{\odot}\right)$, as a function of stellar age.

Convection is assumed to require plate tectonics in order to operate. The reason for this is that plate tectonics helps to cool the planetary interior more efficiently than a stagnant lid configuration (Stevenson 2003). Efficient cooling is required to maintain convection, and is thus essential to keep the action of a magnetic dynamo (Stevenson 1983). The lack of plate tectonics is also suggested as one of the possible reasons for the lack of an intrinsic magnetic field on Venus and Mars (Stevenson et al. 1983; Stevenson 2003). Thus, plate tectonics may be required to keep an Earth-like magnetic dynamo in operation over geological time spans (Lammer et al. 2009).

There are two reasons why the requirement for plate tectonics for convection might be important as far as exoplanets are concerned: (a) For close-in exoplanets, the small orbital distance usually means that the planet is tidally locked (e.g. Grießmeier et al. 2009). It is currently not clear how this influences plate tectonics, but it is sometimes suggested that tidal locking may inhibit plate tectonics partially or completely. (b) For Super-Earth planets, is it still being debated whether plate tectonics is likely to occur or not (Valencia et al. 2007; O'Neill et al. 2007).

Thus, we see that plate tectonics is essential for convection, which is a necessary ingredient for magnetic dynamo action. In the following, we will assume that a magnetic dynamo does exist. However, even if convection is present and a magnetic dynamo exists, the magnetic moment is not necessarily large enough to efficiently shield the planet. The tidally locked state of close-in exoplanets means that they have low rotation rates, which according to scaling laws derived from theoretical considerations - leads to a reduction in the planetary magnetic moment (e.g. Grießmeier et al. 2005) †.

Following the approach of Grießmeier et al. (2005), one finds that for the slowly rotating tidally locked planets, the magnetic moment is much smaller than for freely rotating planets. For example, an Earth-like planet in an orbit of $0.2 \mathrm{AU}$ around a star with 0.5 stellar masses, may have a magnetic moment in the range $0.02 \mathcal{M}_{\mathrm{E}}<\mathcal{M}<0.15 \mathcal{M}_{\mathrm{E}}$ $\left(\mathcal{M}_{\mathrm{E}}\right.$ is the value of Earth's current magnetic moment). In the following, the minimum value will be adopted to estimate an upper limit for atmospheric espace (Sect. 4.1), but for the cosmic ray flux (Sect. 4.2), $\mathcal{M}$ is assued to take the maximum allowed value.

$\dagger$ While the analytical models considered here predict a decrease of magnetic moment with decreasing rotation rate, numerical experiments indicate that the magnetic moment may be independent of the angular frequency. Christensen \& Aubert (2006) and Olson \& Christensen (2006) have studied numerically an extensive set of dynamo models, varying the relevant control parameters by at least two orders of magnitude. In these simulations, the magnetic field is mainly controlled by the buoyancy flux. This is in contradiction with the previously discussed concept. Future studies are required to clarify the relation between magnetic field strength, the planetary rotation rate, electrical conductivity, and buoyancy flux. 
In the following, the magnetosphere is modelled as a cylinder topped by a half-sphere (Voigt 1981; Stadelmann 2005; Grießmeier et al. 2005). The size of the magnetosphere is characterized by the magnetopause standoff distance $R_{s}$, i.e. the extent of the magnetosphere along the line connecting the star and the planet. $R_{s}$ can be obtained from the pressure equilibrium at the substellar point. This pressure balance includes the stellar wind ram pressure, the stellar wind thermal pressure of electrons and protons, and the planetary magnetic field pressure. For a given planetary orbital distance $d$, only the magnetospheric magnetic pressure is a function of the distance to the planet, while the other contributions are constant. Thus, the standoff distance $R_{s}$ is found to be (Grießmeier et al. 2007):

$$
R_{s}=\left[\frac{\mu_{0} f_{0}^{2} \mathcal{M}^{2}}{8 \pi^{2}\left(m n v^{2}+2 n k_{B} T\right)}\right]^{1 / 6} .
$$

Here, $f_{0}=1.16$ is the form factor of the magnetosphere and includes the magnetic field caused by the currents flowing on the magnetopause (Voigt 1995; Grießmeier et al. 2004). From eq. (3) it is evident that a small magnetic moment $\mathcal{M}$, a high stellar wind velocity $v$ or a high stellar wind density $n$ will lead to a small magnetosphere.

\section{Consequences}

The weak magnetic shielding of close-in planets against stellar wind of young stars and against stellar CMEs has important consequences for the planet. The implications that will be discussed in the following are strong atmospheric erosion via stellar wind and CMEs, and the enhanced flux of galactic cosmic rays onto the planetary atmosphere.

\subsection{Atmospheric escape}

Here, we explore the region in parameter space where the stellar wind and CMEs can compress the magnetosphere down to a certain altitude level. In the following we chose the critical altitude level to be equal to $R_{\text {crit }}=1.15 R_{P}=1.15 R_{E}$ (i.e. strong atmospheric loss when the magnetosphere is compressed to less than $0.15 R_{E}=1000 \mathrm{~km}$ above the planetary surface). The numerical study of Lammer et al. (2007, e.g. Figure 9) shows that under certain conditions, the critical altitude level may be as high as $2 R_{E}$ (i.e. 1 $R_{E}$ above the planetary surface), so that this is a conservative assumption.

Fig. 2 shows the region in parameter space where the magnetosphere of an exoplanet with mass, radius, and internal parameters identical to those of the Earth can be strongly compressed (i.e. $R_{s}<R_{\text {crit }}$ ) under the action of strong (= dense) CMEs or under the action of a young stellar wind, respectively. In the tidally locked regime, the magnetic moment was taken to be equal to the minimum value, but tidal locking was assumed only when the timescale for synchronous rotation is < $100 \mathrm{Myr}$ (denoted as "locked"). For timescales between $100 \mathrm{Myr}$ and 10 Gyr ("potentially locked") and for timescales above 10 Gyr ("unlocked") the magnetic moment was estimated using the (larger) initial rotation rate of the planet.

In Fig. 2(a), it can be seen that almost all Earth-like exoplanets within habitable zones (HZs, grey shaded area) of M-stars will experience this extreme magnetospheric compression. In such cases, CMEs bring the magnetosphere down to the level of the ionopause, and the planetary atmospheres can be heavily eroded (Khodachenko et al. 2007; Lammer et al. 2007).

Fig. 2(b) is similar to Fig. 2(a), but it studies the effect of the age-dependent stellar wind rather than that of stellar CMEs. Three cases are considered: The stellar wind of a star of 0.7 Gyr age (all three dotted areas), the stellar wind of a star of 1.0 Gyr age 


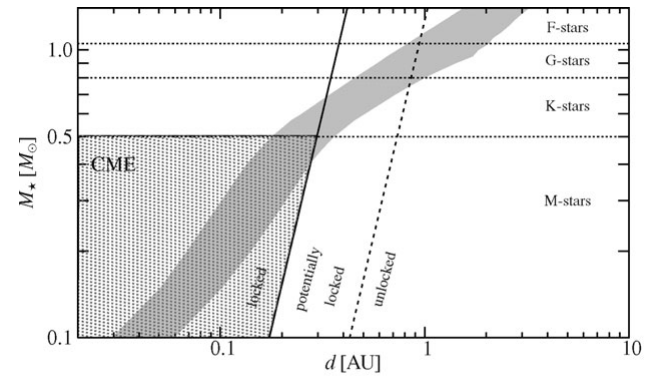

(a) Atmospheric erosion by CMEs.

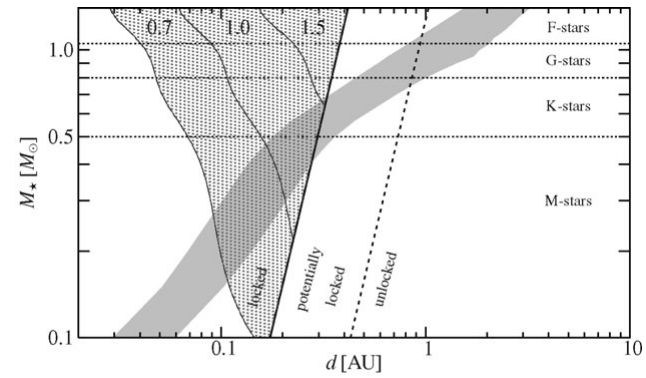

(b) Atmospheric erosion by stellar wind.

Figure 2. Comparison between the HZ (grey shaded area) and the areas where strong magnetospheric compression is possible by CMEs or stellar wind. The dotted area denotes the region where the magnetosphere can be compressed down to 1.15 Earth radii or less (i.e., $1000 \mathrm{~km}$ above the planetary surface). 4.1 Magnetospheric compression by strong CMEs. From Khodachenko et al. (2007). 4.1 Magnetospheric compression by stellar wind of a young star. Three cases are considered: Stellar wind of a star of 0.7 Gyr age (all three dotted areas), Stellar wind of a star of 1.0 Gyr age (upper two dotted areas), Stellar wind of a star of 1.5 Gyr age (top dotted area only).

(top two dotted areas), and the stellar wind of a star of 1.5 Gyr age (upper dotted area only). One can clearly see that for a young star (0.7 Gyr), all planets in a habitable zone between 0.1 and $\sim 0.3$ AU have magnetospheric altitudes lower than the critical altitude level, so that strong erosion is likely. As the star's age increases, the stellar wind becomes more tenuous, and the region of strong erosion in the $d-M_{\star}$-parameter space shrinks.

An additional effect of stellar age should be mentioned: Not only is the magnetosphere of a close-in planet around a young star strongly compressed, but also the planetary atmosphere is more extended due to the intense heating by the high stellar X-ray and EUV-flux (Lammer et al. 2003; Grießmeier et al. 2004; Ribas et al. 2005). Thus, the critical level the magnetosphere is allowed to reach, $R_{\text {crit }}(t)$, in reality is age-dependent, and it is much more likely for a young planetary magnetosphere to reach the critical altitude than one would find using present day stellar irradiation.

From Figures 2(a) and 2(b) it becomes clear that the relatively weak intrinsic magnetic fields of tidally locked planets are not suffient to protect Earth-like exoplanets against significant atmospheric erosion caused by CMEs and the stellar wind. The sharp cutoff at the right edge of the dotted area demonstrates the importance of magnetic shielding: Within the dotted area, planets are tidally locked. They are assumed to have a small magnetic moment, and magnetic protection is weak. To the right of the dotted area however, the planets are assumed to be freely rotating, so that they can maintain a much higher magnetic moment and are well protected against strong atmospheric erosion.

\subsection{Cosmic ray flux}

In order to quantify the protection of extrasolar Earth-like planets against galactic cosmic rays, the motion of galactic cosmic protons through magnetospheres of extrasolar planets has been investigated numerically (Grießmeier et al. 2005, 2009). From these calculations, one finds that the cosmic ray flux is strongly enhanced in the case of a weakly magnetized planet. Fig. 3 shows the reference energy spectrum outside the magnetosphere (Seo et al. 1994) as a dash-dotted line. The energy spectrum at the of the Earth's atmosphere is shown as a solid line, and the cosmic ray energy spectrum for a weakly magnetized Earthlike exoplanet in an orbit of $0.2 \mathrm{AU}$ around a $0.5 M_{\odot} \mathrm{K} / \mathrm{M}$ star is shown as a dashed line. Due to tidal locking, the magnetic moment of that planet is reduced to (at most) $15 \%$ 


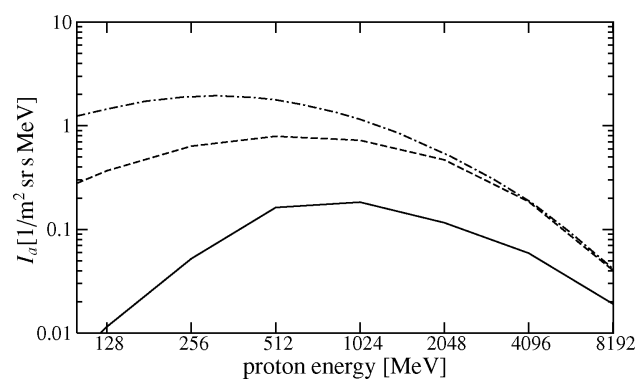

Figure 3. Dash-dotted line: energy spectrum outside the magnetosphere. Dashed line: energy spectrum of cosmic ray protons impacting the atmosphere $(100 \mathrm{~km}$ above the surface) of an Earth-like exoplanet at $0.2 \mathrm{AU}$ around a $0.5 \mathrm{M} \mathrm{K} / \mathrm{M}$ star. Solid line: energy spectrum of cosmic rays impacting the atmosphere of the Earth. From Grießmeier et al. (2005).

of the Earth's magnetic moment (Grießmeier et al. 2009, section 2.2), which translates to a strongly enhanced cosmic ray flux to the planetary atmosphere.

The increased flux of cosmic ray protons of galactic origin to the upper atmospheres of M-star planets has implications for the flux of (secondary) cosmic ray particles reaching the ground, which can be expected to have biological implications. It also influences the atmospheric composition and thus the remote detection of biomarkers as well as the surface UV flux.

\subsubsection{Direct biological impact}

While a quantitative treatment is not yet available, the increased flux of cosmic rays to the top of the planetary atmosphere has implications for the flux of secondary radiation to the surface. When galactic cosmic rays of sufficiently high energy reach the planetary atmosphere, they generate showers of secondary cosmic rays. Of the different components of such showers, slow neutrons have the strongest influence on biological systems. The propagation of high energy cosmic ray particles also depends on the composition and density of the planetary atmosphere. For an Earth-like atmosphere, the minimum energy which a proton must have to initiate a nuclear interaction that is detectable at sea level is approximately $450 \mathrm{MeV}$ (Reeves et al. 1992; Shea \& Smart 2000). Terrestrial planets with dense atmospheres like Venus (100 bar surface pressure) would be better shielded by the planetary atmosphere, so that no secondary radiation can reach the surface. On the other hand, for planets with thin atmospheres like Mars (6 mbar surface pressure), the surface would probably be totally sterilised, even for a relatively low cosmic ray flux.

Keeping the atmospheric pressure constant at $\sim 1$ bar, the flux at energies $>450 \mathrm{MeV}$ is strongly increased for a weakly magnetized planet (see Fig. 3), and a large increase of secondary cosmic rays can be expected at the planetary surface.

Biological effects, namely an increase in cell fusion indices for different cell-lines, were found to be significantly correlated with the neutron count rate at the Earth's surface (Belisheva et al. 2005; Grießmeier et al. 2005). Similar, but much stronger and more diverse effects were observed during large solar particle events, where solar cosmic rays dominate over galactic cosmic rays. The effect of cosmic rays on cells and on DNA are potentially hazardous for life. On the other hand, changes at the genetic level are a necessary condition for biological evolution, so that cosmic rays may also play a role in radiation-induced evolutionary events.

As tidally locked Earth-like exoplanets inside the $\mathrm{HZ}$ of $\mathrm{K} / \mathrm{M}$ stars are only weakly protected against high energetic cosmic rays, they can be expected to experience a higher surface neutron flux and stronger biological effects than Earth-like planets with a strong 
magnetic field. For this reason, it may be more difficult for life to develop on the surface of planets around such stars.

\subsubsection{Atmospheric modification}

The influence of a high flux of galactic cosmic rays (GCRs) and of stellar energetic particles on the atmospheres of planets in the HZ around M-stars is studied by Grenfell et al. (2007) and Grenfell et al. (2010). When cosmic ray particles travel through an Earth-like atmosphere, they have sufficient energy to break the strong $\mathrm{N}_{2}$ molecule, and the chemical products react with oxygen to form nitrogen oxides $\left(\mathrm{NO}_{\mathrm{x}}\right)$. If UV levels are intense, e.g. corresponding to the upper mesosphere and above on the Earth, then the $\mathrm{NO}_{\mathrm{x}}$ cannot survive. $\mathrm{NO}_{\mathrm{x}}$ affects biomarkers differently depending on altitude. In a stratospheric environment, $\mathrm{NO}_{\mathrm{x}}$ depletes ozone by catalytic cycles (Crutzen 1970). Once ozone is affected, the other biomarkers can change too, because ozone affects the temperature profile (hence e.g. mixing processes, chemical sources and sinks) and UV levels (hence photolysis rates) for other chemical species.

This is important, because atmospheres of extrasolar planets will be studied from the point of view of possible biological activity in the near future. "Atmospheric biomarkers" are compounds present in an atmosphere which imply the presence of life and which cannot be explained by inorganic chemistry alone. The simultaneous presence of significant amounts of atmospheric reducing gases (e.g. methane) and oxidising gases (e.g. oxygen) as on the Earth is also an indicator of biological activity (Lovelock 1965; Sagan et al. 1993). Good biomarkers include oxygen (produced by photosynthesis), ozone (mainly produced from oxygen) and nitrous oxide (produced almost exclusively from bacteria). For the study of biomarkers in planetary atmospheres, it is important to know all inorganic effects which can reduce the abundances of these molecules. Modelling studies are required to rule out false conclusions in cases where inorganic chemistry, e.g. reactions triggered by the influx of cosmic ray particles, can mimic (or mask) the presence of life.

Using the cosmic ray spectrum of Fig. 3, Grenfell et al. (2007) found that for Earthlike planets in close orbits around M-stars, GCRs may only change the abundances of biomarker molecules (especially water and ozone) by a small factor. This can also be seen by comparing the cases "Earth GCR" and "Exoplanet GCR" in Fig. 4. The case is different for solar energetic particles (Grenfell et al. 2010), which can reduce the abundance of ozone in the planetary atmosphere by several orders of magnitude. This effect can be seen in Fig. 4, where "Exoplanet SPE" shows the effect of solar energetic particles (adapted from Grenfell et al. 2010). Such changes in atmospheric biomarker concentrations have to be taken into account when searching for biosignatures in the spectra of Earth-like exoplanets by future space missions like DARWIN (Fridlund 2004) or SEE-COAST (Schneider et al. 2006).

\subsubsection{Modification of $U V$ shielding}

Both direct UV radiation (Buccino et al. 2007; Scalo et al. 2007) and indirect UV radiation generated by energetic particles (Smith et al. 2004) can have important consequences for biogenic processes on M-star planets. As was shown in Section 4.2.2, the planetary ozone layer of a weakly magnetized planet can be virtually destroyed during a solar energetic particle event. The corresponding enhancement of the UV flux has to be taken into account when biological effects of UV radiation are considered. The influence of UV radiation of different wavelenth on life is also discussed by Cuntz et al. (2010). 


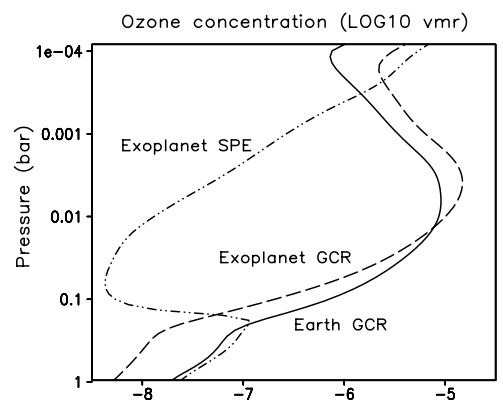

Figure 4. Atmospheric biomarker profile concentrations (log volume mixing ratio): ozone for three different cases (see text). From Grenfell et al. (2010).

\section{Conclusions}

Because of tidal locking, extrasolar planets with orbits in the habitable zone of $\mathrm{K} / \mathrm{M}$ are likely to have much smaller magnetic moments than more distant planets. Combined with the strong ram pressure of dense and fast stellar winds and the high CME activity expected around young stars, this leads to the conclusion that such planets will have small magnetospheres which offer only limited magnetic protection.

The weak magnetic protection may lead to strong atmospheric erosion during the early stages of stellar evolution, which could be strong enough to erode the atmosphere or the planets' water inventory via non-thermal atmospheric loss processes. Another consequence of the weak magnetic protection is a high flux of galactic cosmic rays and stellar energetic particles to the planetary atmosphere. This has implications for potential habitability, but also for the atmospheric chemistry and composition (e.g. destruction of atmospheric ozone). These effects have also to be considered for missions studying biosignatures in the observed spectra of Earth-like exoplanets.

Overall, the magnetic protection of exoplanets in the habitable zone of $\mathrm{K} / \mathrm{M}$ stars can be weak, so that these planets may only be weakly shielded against stellar activity.

\section{Acknowledgements}

This study was supported by the International Space Science Institute (ISSI) through the ISSI Team "Evolution of Exoplanet Atmospheres and their Characterisation" and by the Helmholtz association through the research alliance "Planetary Evolution and Life". MK acknowledges the Austrian Fond zur Förderung der wissenschaftlichen Forschung (project P21197-N16).

\section{References}

Beaulieu, J.-P., Bennett, D. P., Fouqué, P., et al. 2006, Nature, 439, 437

Belisheva, N. K., Kuzhevskii, B. M., Vashenyuk, E. V., \& Zhirov, V. K. 2005, Doklady Biochemistry and Biophysics, 402, 254

Bouchy, F., Mayor, M., C., et al. 2009, Astron. Astrophys., 496, 527

Buccino, A. P., Lemarchand, G. A., \& Mauas, P. J. D. 2007, Icarus, 192, 582

Christensen, U. R. \& Aubert, J. 2006, Geophys. J. Int., 166, 97

Crutzen, P. J. 1970, Q. J. R. Meteorol. Soc., 96, 320

Cuntz, M., Guinan, E. F., \& Kurucz, R. L. 2010, this proceedings, p. 419

Forveille, T., Bonfils, X., Delfosse, X., et al. 2009, Astron. Astrophys., 493, 645

Fridlund, C. V. M. 2004, Adv. Space Res., 34, 613

Grenfell, J. L., Grießmeier, J.-M., Patzer, B., et al. 2007, Astrobiology, 7, 208

Grenfell, J. L., Grießmeier, J.-M., Patzer, B., et al. 2010, to be submitted 
Grießmeier, J.-M., Preusse, S., Khodachenko, M., et al. 2007, Planet. Space Sci., 55, 618

Grießmeier, J.-M., Stadelmann, A., Grenfell, J. L., Lammer, H., \& Motschmann, U. 2009, Icarus, 199,526

Grießmeier, J.-M., Stadelmann, A., Motschmann, U., et al. 2005, Astrobiology, 5, 587

Grießmeier, J.-M., Stadelmann, A., Penz, T., et al. 2004, Astron. Astrophys., 425, 753

Houdebine, E. R., Foing, B. H., \& Rodonò, M. 1990, Astron. Astrophys., 238, 249

Howard, A. W., Johnson, J. A., Marcy, G. W., et al. 2009, Astrophys. J., 696, 75

Kasting, J. F., Whitmire, D. P., \& Reynolds, R. T. 1993, Icarus, 101, 108

Khodachenko, M. L., Ribas, I., Lammer, H., et al. 2007, Astrobiology, 7, 167

Lammer, H., Bredehöft, J. H., Coustenis, A., et al. 2009, Astron. Astrophys. Rev., 17, 181

Lammer, H., Lichtenegger, H. I. M., Kulikov, Y. N., et al. 2007, Astrobiology, 7, 185

Lammer, H., Selsis, F., Ribas, I., et al. 2003, Astrophys. J., 598, L121

Lovelock, J. E. 1965, Nature, 207, 568

Lovis, C., Mayor, M., Pepe, F., et al. 2006, Nature, 441, 305

Mann, G., Jansen, F., MacDowall, R. J., Kaiser, M. L., \& Stone, R. G. 1999, Astron. Astrophys., 348,614

Mayor, M., Udry, S., Lovis, C., et al. 2009, Astron. Astrophys., 493, 639

Newkirk, Jr., G. 1980, in The Ancient Sun: Fossil Record in the Earth, Moon and Meteorites, ed. R. O. Pepin, J. A. Eddy, \& R. B. Merrill, 293-320

Olson, P. \& Christensen, U. R. 2006, Earth Planet. Sci. Lett., 250, 561

O'Neill, C., Jellinek, A. M., \& Lenardic, A. 2007, Earth Planet. Sci. Lett., 261, 20

Parker, E. N. 1958, Astrophys. J., 128, 664

Prölss, G. W. 2004, Physics of the Earth's Space Environment (Berlin: Springer-Verlag)

Reeves, G. D., Cayton, T. E., Gary, S. P., \& Belian, R. D. 1992, J. Geophys. Res., 97, 6219

Ribas, I., Font-Ribera, A., \& Beaulieu, J.-P. 2008, Astrophys. J., 677, L59

Ribas, I., Guinan, E. F., Güdel, M., \& Audard, M. 2005, Astrophys. J., 622, 680

Rivera, E. J., Lissauer, J. J., Butler, R. P., et al. 2005, Astrophys. J., 634, 625

Sagan, C., Thomson, W. R., Carlson, R., Gurnett, D., \& Hord, C. 1993, Nature, 365, 715

Scalo, J., Kaltenegger, L., Segura, A., et al. 2007, Astrobiology, 7, 85

Schneider, J., Riaud, P., Tinetti, G., et al. 2006, in SF2A-2006: Semaine de l'Astrophysique Francaise, ed. D. Barret, F. Casoli, T. Contini, G. Lagache, A. Levacelier, \& L. Pagani, 429-432

Seo, E. S., McDonald, F. B., Lal, N., \& Webber, W. R. 1994, Astrophys. J., 432, 656

Shea, M. A. \& Smart, D. F. 2000, Space Sci. Rev., 93, 187

Smith, D. S., Scalo, J., \& Wheeler, J. C. 2004, Icarus, 171, 229

Stadelmann, A. 2005, PhD thesis, Technische Universität Braunschweig, ISBN 3936586-42-X, Copernicus-GmbH Katlenburg-Lindau, URL: http://www.digibib.tubs.de/?docid $=00000002$

Stevenson, D. J. 1983, Rep. Prog. Phys., 46, 555

Stevenson, D. J. 2003, Earth Planet. Sci. Lett., 208, 1

Stevenson, D. J., Spohn, T., \& Schubert, G. 1983, Icarus, 54, 466

Tarter, J. C., Backus, P. R., Mancinelli, R. L., et al. 2007, Astrobiology, 7, 30

Udry, S., Bonfils, X., Delfosse, X., et al. 2007, Astron. Astrophys., 469, L43

Valencia, D., O'Connell, R. J., \& Sasselov, D. D. 2007, Astrophys. J., 670, L45

Voigt, G.-H. 1981, Planet. Space Sci., 29, 1

Voigt, G.-H. 1995, in Handbook of atmospheric electrodynamics, ed. H. Volland, Vol. II (Boca Raton: CRC Press), 333-388

Wood, B. E. 2004, Living Rev. Solar Phys., 1, 2, URL: http://www.livingreviews.org/lrsp-20042, accessed on 23 February 2007

Wood, B. E., Müller, H.-R., Zank, G. P., \& Linsky, J. L. 2002, Astrophys. J., 574, 412

Wood, B. E., Müller, H.-R., Zank, G. P., Linsky, J. L., \& Redfield, S. 2005, Astrophys. J., 628, L143 